Revue des patrimoines

$34 \mid 2018$

Lieux de pouvoirs. Architectures administratives dans la France contemporaine, 1945-2013. Avant/Après la décentralisation

\title{
La construction des cités administratives en France durant les « Trente Glorieuses » à travers les sources des Archives nationales
}

The building of administrative cities during the thirty years of post-war prosperity, sources at the national archives

Pascal Riviale

\section{(2) OpenEdition}

1 Journals

Édition électronique

URL : http://journals.openedition.org/insitu/15679

DOI : 10.4000/insitu. 15679

ISSN : 1630-7305

Éditeur

Ministère de la culture

Référence électronique

Pascal Riviale, «La construction des cités administratives en France durant les « Trente Glorieuses » à travers les sources des Archives nationales », In Situ [En ligne], 34 | 2018, mis en ligne le 04 mai 2018, consulté le 02 mai 2019. URL : http://journals.openedition.org/insitu/15679 ; DOI : 10.4000/ insitu. 15679

Ce document a été généré automatiquement le 2 mai 2019.

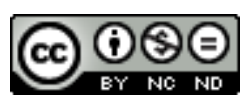

In Situ Revues des patrimoines est mis à disposition selon les termes de la licence Creative Commons Attribution - Pas d'Utilisation Commerciale - Pas de Modification 4.0 International. 


\title{
La construction des cités
} administratives en France durant les « Trente Glorieuses » à travers les sources des Archives nationales

\author{
The building of administrative cities during the thirty years of post-war \\ prosperity, sources at the national archives
}

Pascal Riviale

1 Après la Seconde Guerre mondiale, dans un contexte de reconstruction massive puis de fort développement de l'urbanisation, et du fait de l'évolution des pratiques de gestion des services publics, la construction de cités administratives occupe une place prépondérante dans le paysage architectural et urbanistique de la France des «Trente Glorieuses ». Les archives des services des Bâtiments civils et de l'Urbanisme conservées aux Archives nationales permettent de documenter cette page de l'histoire architecturale, illustrant à la fois les ambitions, les tâtonnements voire les tergiversations des divers acteurs - tant au niveau local que national - quant à la conception de ces bâtiments. Les besoins importants en matière de construction dans les années qui suivent la guerre exercent une pression notable tant sur les collectivités locales que sur les divers niveaux de l'administration départementale ou nationale: les dommages provoqués par le conflit mondial nécessitent parfois de grandes opérations de reconstruction (qu'il s'agisse d'habitat ou de services publics); en outre, la reprise des activités économiques et sociales occasionne un fort développement du tissu urbain à l'échelle nationale, d'où l'émergence de besoins nouveaux en termes de gestion administrative. Très vite va être formulée l'idée de regrouper les administrations en un seul lieu afin d'obtenir une meilleure efficacité et d'offrir un meilleur service aux administrés ${ }^{1}$. Cette idée générique, pour toute rationnelle qu'elle semble, va toutefois se trouver gênée dans son application sur le terrain par diverses contraintes : besoins urgents mais contradictoires, tissu urbain difficile à modifier en centre-ville, manque de crédits, etc. Si les diverses entités administratives impliquées dans ces projets tentent généralement de s'accorder, les 
impératifs budgétaires ou réglementaires, les pressions ou les ambitions locales, comme les choix politiques les emmènent parfois dans des directions divergentes. On entend montrer, dans cet article, à travers les archives des services centraux de l'Architecture, les ambitions, les tiraillements mais aussi les déceptions qui ont émaillé nombre de ces projets de l'après-guerre. Nous nous appuierons essentiellement sur trois exemples (Nancy, Bordeaux et Lyon) qui, sans prétendre à l'exhaustivité, illustrent un certain nombre de situations représentatives de cette page d'histoire de la création architecturale mais aussi de ses vicissitudes.

2 Il paraît nécessaire avant toute chose de présenter les différents acteurs qui sont intervenus dans ces projets architecturaux. À l'échelon central, les principaux protagonistes étaient la Commission centrale de contrôle des opérations immobilières (CCCOI), le ministère de la Reconstruction et de l'Urbanisme et enfin la direction de l'Architecture. La Commission centrale de contrôle des opérations immobilières avait été créée par décret du 2 novembre $1945^{2}$ en vue de rationaliser les divers projets de constructions publiques envisagés sur l'ensemble du territoire national. De par sa position centrale, elle était censée avoir une vue d'ensemble sur ces projets, en évaluer la cohérence et la faisabilité, tant d'un point de vue urbanistique qu'administratif et budgétaire. Dans l'immédiat après-guerre, le ministère de la Reconstruction et de l'Urbanisme joua aussi un rôle majeur dans la construction de bâtiments administratifs, dans la mesure où il fut fréquemment sollicité pour réhabiliter dans l'urgence des édifices affectés - souvent faute de mieux - aux services publics en pleine reconstitution. Enfin, la direction de l'Architecture était l'héritière du service des Bâtiments civils et Palais nationaux (BCPN) fondé au tout début du XIX ${ }^{e}$ siècle. Cette administration, relevant - au cours de la période envisagée ici, soit 1945-1975 - du ministère de l'Éducation nationale puis des Affaires culturelles, comptait plusieurs bureaux chargés de suivre différentes étapes du processus architectural: bureau des Bâtiments civils, bureau de la Construction, bureau des Contrôles, bureau de la Liquidation des dépenses. Ce service était associé depuis ses origines à un Conseil général des bâtiments civils, instance chargée d'évaluer la qualité architecturale des projets de constructions publiques qui lui étaient soumis. Au niveau local, les acteurs concernés au premier chef étaient le cabinet du maire, celui du préfet et les services départementaux de l'Architecture et de l'Équipement, ainsi que la commission départementale des opérations immobilières qui relayait à l'échelon central les dossiers qu'elle avait validés.

\section{Nancy : I'héritage militaire}

3 À l'instar de nombreuses autres villes de France dans les années d'après-guerre ${ }^{3}$, les services publics en quête de locaux à Nancy furent installés à partir de 1949 dans de vieux bâtiments situés dans une annexe de la caserne Thiry, dans le centre-ville, parfois de manière assez précaire et inconfortable. Dans quelques cas, les bâtiments étaient trop endommagés pour être investis tels quels et nécessitaient des travaux importants: "Avant l'installation des services américains dans une partie de la caserne Thiry, le bâtiment $\mathrm{H}$ était sérieusement sinistré tant par faits de guerre que par l'incendie et c'était pour ce motif que dans le projet de cité administrative établi, il devait être entièrement démoli ${ }^{4}$.» On préféra pourtant envisager sa réhabilitation. Lors d'une séance de la Commission centrale de contrôle des opérations immobilières de janvier 1951, on évoquait les contraintes inhérentes à l'exploitation d'édifices qui n'avaient pas été prévus 
à l'origine pour cette nouvelle affectation : «Le Préfet a justifié ces décisions en attirant l'attention sur la situation spéciale de bâtiments militaires transformés qui ne sauraient être utilisés comme des bâtiments neufs [...]. En bref, on tente d'utiliser au mieux avec des transformations les moins coûteuses tout ce qui existe mais il ne peut évidemment être question d'appliquer à de tels bâtiments les normes des constructions neuves $»^{5}$.

Si l'urgence était de trouver des locaux susceptibles d'accueillir ces administrations, on ne perdait néanmoins pas de vue la perspective d'une implantation rationalisée des différents services publics de la ville, impliquant de ce fait nécessairement la construction de nouveaux corps de bâtiments selon un plan d'ensemble dûment réfléchi. Comme le répondrait en 1959 le préfet du département à une demande de construction ponctuelle pour le service de la Navigation, « une telle cité ne peut s'édifier que conformément à un plan de regroupement et suivant un plan masse conçu et approuvé avant toute réalisation $»^{6}$. Aussi, au cours des années suivantes, de nouvelles études furent commandées, achoppant cependant sur des questions d'accès et de faible disponibilité des espaces constructibles dans cette partie centrale de la ville de Nancy. Entre temps, par arrêté du 9 juillet 1956, la cité administrative de Nancy, « ancienne annexe de la caserne Théry [sic] $]^{7}$ » était classée parmi les bâtiments civils de l'État; si les précédents travaux avaient été assurés par le ministère de la Reconstruction et de l'Urbanisme, l'entretien, les aménagements et constructions à venir seraient désormais à la charge de l'administration des Bâtiments civils. Jean Bourgon (1895-1959), architecte des Bâtiments civils et Palais nationaux établi à Nancy, était déjà sollicité depuis quelques années afin de suivre les travaux d'entretien ainsi que les projets de construction relevant de cette administration ${ }^{8}$.

Figure 1

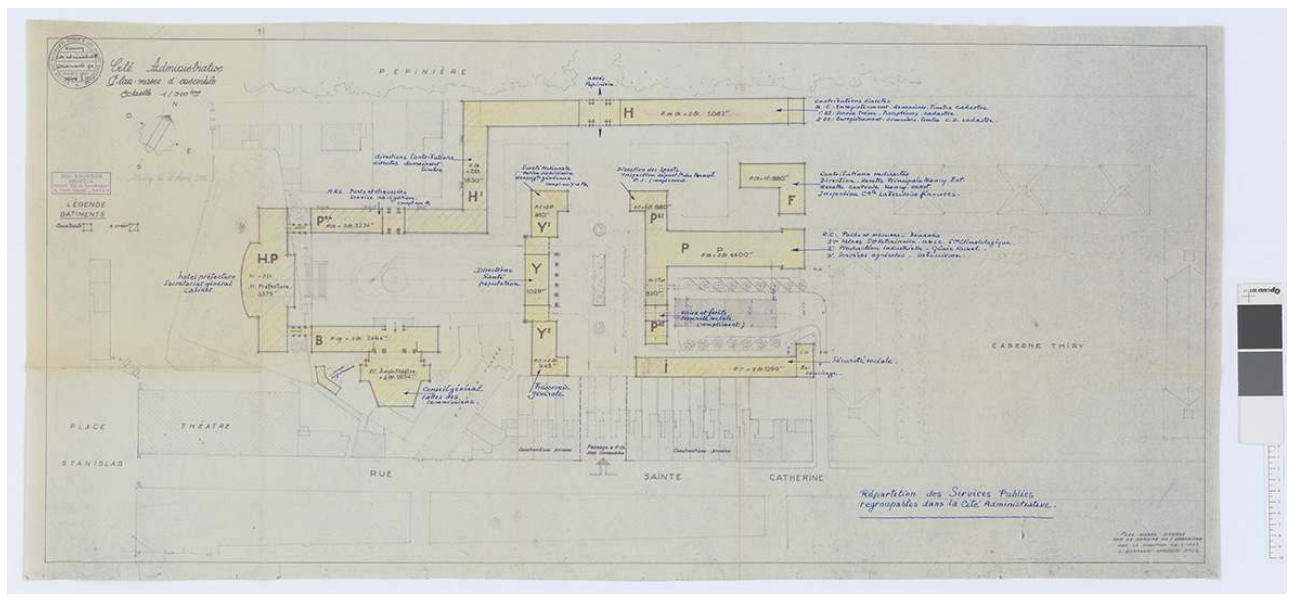

Cité administrative de Nancy. Plan-masse d'ensemble du projet présenté en 1956 par l'architecte Jean Bourgon.

(c) Archives nationales, 19880557/64

En 1957, lors d'une nouvelle réunion de la commission départementale de contrôle des opérations immobilières, il présenta un nouveau projet pour la cité, reposant sur la construction de deux immeubles de quatre étages après arasement d'une série de bâtiments vétustes et de faibles dimensions (fig. 1) :

J'ai donc cherché la possibilité de construire dans les emplacements laissés libres dans le périmètre de la cité administrative actuelle rue Sainte-Catherine [...]. L'architecture des nouveaux bâtiments serait traitée autrement que dans le style 
« caserne » et petit à petit suivant les crédits disponibles, les façades des bâtiments actuels pourraient être reprises et transformées dans un sens plus agréable et plus esthétique9. une ville alors en pleine expansion. Aussi, le préfet adressa-t-il en 1962 à la Commission centrale de contrôle des opérations immobilières un contre-projet radicalement différent, ayant davantage la faveur des collectivités locales: la construction de nouveaux bâtiments sur un terrain nettement plus vaste, situé à la périphérie de la ville. Le projet formulé en fait semble-t-il dès février 1961 par l'architecte Paul La Mache (1918-1999) prévoyait une tour en étoile à quatre branches comportant vingt-sept niveaux, ainsi que plusieurs bâtiments annexes (halls, cantine, crèche) et un grand parking ${ }^{10}$. L'argument principal pour l'abandon des annexes de la caserne résidait dans l'exiguïté du terrain constructible dans ce quartier, certes central mais déjà très densément urbanisé et présentant de lourdes contraintes patrimoniales. Comme le résumait l'inspecteur général des BCPN (et ingénieur des Arts et Manufactures) Jean Démaret (1897-1967) dans son rapport remis au Conseil général des bâtiments de France :

On a projeté d'édifier la nouvelle cité sur un terrain de plus de quatre hectares situé boulevard d'Haussonville à $2 \mathrm{~km}$ du centre de Nancy dans un quartier en voie de développement et où sont en construction : l'université, les logements, etc. Terrain très bien desservi par plusieurs boulevards et lignes d'autobus. Ce terrain est actuellement propriété de la ville [...]. La ville de Nancy et le Conseil général de Meurthe-et-Moselle sont très favorables à la construction de cette nouvelle cité administrative dans un quartier nouveau. On estime en effet que cette construction importante sera un catalyseur dans une zone en plein développement et favorisera l'extension de ce quartier ${ }^{11}$.

Dans ses conclusions, l'inspecteur Démaret penchait plutôt pour cette nouvelle solution. Pourtant, la Commission centrale de contrôle des opérations immobilières persista dans son opposition, faisant valoir que le terrain d'Haussonville était trop éloigné du centreville et de la préfecture. Elle convenait dans le même temps que les annexes de la caserne Thiry étaient trop vétustes pour être fonctionnelles; elle déclarait par conséquent «qu'après acquisition et arasement des immeubles vétustes situés en bordure de la rue Sainte-Catherine, le terrain serait suffisamment vaste pour permettre l'édification par tranches d'une cité administrative répondant aux besoins, les bâtiments actuels étant progressivement démolis $»^{12}$. Cette position allait à l'encontre des pouvoirs locaux et ne tenait pas compte des préconisations de l'administration des Monuments historiques. De fait, dans une note adressée en novembre 1966 au président de la CCCOI, André Coumet, sous-directeur des Monuments historiques et des Sites, rapportait l'avis de la Commission supérieure des Monuments historiques (section des abords), qui avait « estimé que, au cas où le projet serait repris, il ne pourrait l'être qu'aux conditions suivantes: la hauteur maximum des immeubles à construire ne devrait pas dépasser $\mathrm{R}+4$ au centre du terrain, et $\mathrm{R}+2$ en bordure du parc; les immeubles bordant la rue Sainte-Catherine ne devraient pas être démolis $»^{13}$.

En vérité, le projet ne fut pas totalement abandonné mais réorienté dès l'année suivante.

Le préfet et la CCCOI travaillaient maintenant à un double projet, puisque

en définitive, l'importance de la ville de Nancy et les perspectives de développement de l'agglomération justifiaient la réalisation de deux centres administratifs principaux: l'un, correspondant à la cité administrative déjà aménagée dans l'ancienne annexe de la caserne Thiry et permettant de regrouper dans des conditions améliorées les services recevant le plus de public et le plus en 
contact avec la préfecture tant que celle-ci sera maintenue à son emplacement actuel, l'autre, sur le terrain du boulevard d'Haussonville, destiné à assurer la réinstallation de services techniques, de services lourds ou de services recevant le moins de public ${ }^{14}$.

Ce n'était qu'une déclaration de principe puisque le programme n'était pas complètement budgété. En outre, les désaccords et tergiversations des différents acteurs ne pouvaient que ralentir la bonne marche des choses. Dans une note adressée au directeur de l'Architecture en juin 1968, Yves Lormant, administrateur civil, faisait part des tensions qu'il avait ressenties lors d'une récente réunion à la préfecture, à Nancy, le directeur départemental de l'Équipement étant allé jusqu'à exprimer clairement ses doutes quant à l'intérêt même du projet :

Les discussions ont été difficiles et pénibles car dominées par la virulence, parfois discourtoise, des attaques de M. Laure [le directeur départemental de l'Équipement], à l'égard de M. Lamoise [l'architecte des Bâtiments civils en charge $\mathrm{du}$ projet]. En ce qui concerne l'utilité d'une cité administrative à Nancy, j'ai rappelé au directeur de l'Équipement que la décision d'édifier des cités était une décision gouvernementale et que l'opportunité d'en construire une à Nancy avait été reconnue par la CCCOI. Répondant à ses doutes quant à la nécessité de ces cités dans trente ans, M. le préfet et moi-même avons souligné que cette perspective était trop éloignée pour affirmer qu'alors l'administration n'aurait plus besoin de locaux et qu'en tout état de cause, les grands besoins immédiats devaient être réglés [...]. M. le préfet a été très net et a précisé à $M$. Laure que le choix d'Haussonville était définitif et n'avait pas à être remis en question. En ce qui concerne le parti architectural proposé, et l'environnement, M. Laure a fait des critiques virulentes [...]. M. Lamoise ayant dit que le parti proposé était l'aboutissement de nombreuses années d'études, j'ai indiqué qu'il n'était pas dans les habitudes de nos architectes de présenter plusieurs projets au Conseil général des bâtiments de France en laissant à celui-ci la responsabilité du choix. M. Lamoise a, d'ailleurs, ajouté que, bien sûr, il accepterait des remarques faites par des confrères, mais se refusait à reprendre des études complètement différentes sous la pression d'un profane voulant lui imposer un «Sarcelles » administratif. Si tel était le cas, il préférerait abandonner tout de suite l'affaire car il estimait que l'architecte devait rester libre de sa conception ${ }^{15}$.

10 Max Querrien, directeur de l'Architecture, ayant lui-même exprimé ses réticences sur les qualités formelles et méthodologiques $\mathrm{du}$ projet de Roger Lamoise, le directeur départemental de l'Équipement suggéra une solution alternative :

Comme suite à la réunion de mercredi dernier, je me permets de vous faire parvenir, ci-joint, deux photos de réalisations d'Henri Prouvé : l'immeuble Joffre Saint-Thiébaut, au centre de Nancy; le laboratoire régional exécuté pour notre compte, à Tomblaine dans la périphérie de Nancy. Ce qui me séduit chez Henri Prouvé, c'est, non seulement son talent, tel qu'il s'exprime dans des immeubles comme le Joffre Saint-Thiébaut ou le laboratoire régional, mais aussi son extrême modestie à l'égard des programmes, son esprit de recherche poussée, enfin son rayonnement personnel ${ }^{16}$.

11 C'est finalement un autre architecte nancéien, Pierre Sirvin, qui fut contacté, et dans les derniers jours de l'année 1968 un nouvel arrêté ministériel était pris, chargeant conjointement Lamoise et Sirvin de reprendre le projet sur de nouvelles bases ${ }^{17}$. En 1969 , la direction de l'Équipement diligentait une étude sur l'environnement urbanistique du quartier d'Haussonville auprès $d u$ cabinet B.E.R.U. afin de mesurer l'impact de l'implantation de cette future cité administrative tandis que Pierre Sirvin adressait ses premières études. On aurait pu croire que le projet était enfin bien lancé, pourtant en juin 1972 le ministère des Affaires culturelles (par l'entremise de son sous-directeur de la 
Création architecturale et des Constructions publiques Jean Jenger) adressait une note au secrétaire d'État auprès du Premier ministre afin de lui exposer la difficulté de programmation à Nancy d'un double projet qui n'était toujours pas entièrement budgété :

Le projet n'a pu encore être retenu dans le programme financé sur le chapitre des charges communes, si bien que le préfet semble disposé à promouvoir des opérations partielles [...]. Ces opérations indépendantes et dispersées risquent de ne pouvoir s'intégrer à terme dans un projet d'ensemble [...]. Le risque de voir une réalisation, même très partielle, bloquer ultérieurement une opération globale, me conduit à vous proposer d'organiser une réunion de l'ensemble des services intéressés par la cité administrative de Nancy. Même si l'état ne peut prendre en charge l'ensemble de l'opération dans les prochaines années, une telle réunion aurait pour intérêt de définir une méthode qui permettrait au préfet d'entreprendre dans l'immédiat certaines réalisations partielles, sans pour autant compromettre les chances d'une opération d'ensemble dans un avenir reculé18.

Ces nouvelles démarches ne paraissent ne pas avoir eu plus de succès. Enfin, en 1995, une restructuration du site de Sainte-Catherine fut une nouvelle fois envisagée, mais ce projet - présenté parmi les programmes du concours Europan - ne vit pas plus le jour. Actuellement, les anciens locaux aménagés à proximité de la caserne Thiry sont toujours là et le sujet de la nouvelle cité prévue dans le quartier d'Haussonville semble avoir été abandonné ${ }^{19}$; seul le centre d'affaires - dont la construction au voisinage de la cité administrative avait été planifiée dans les années 1960 -, y a été réalisée ${ }^{20}$ (fig. 2).

Figure 2

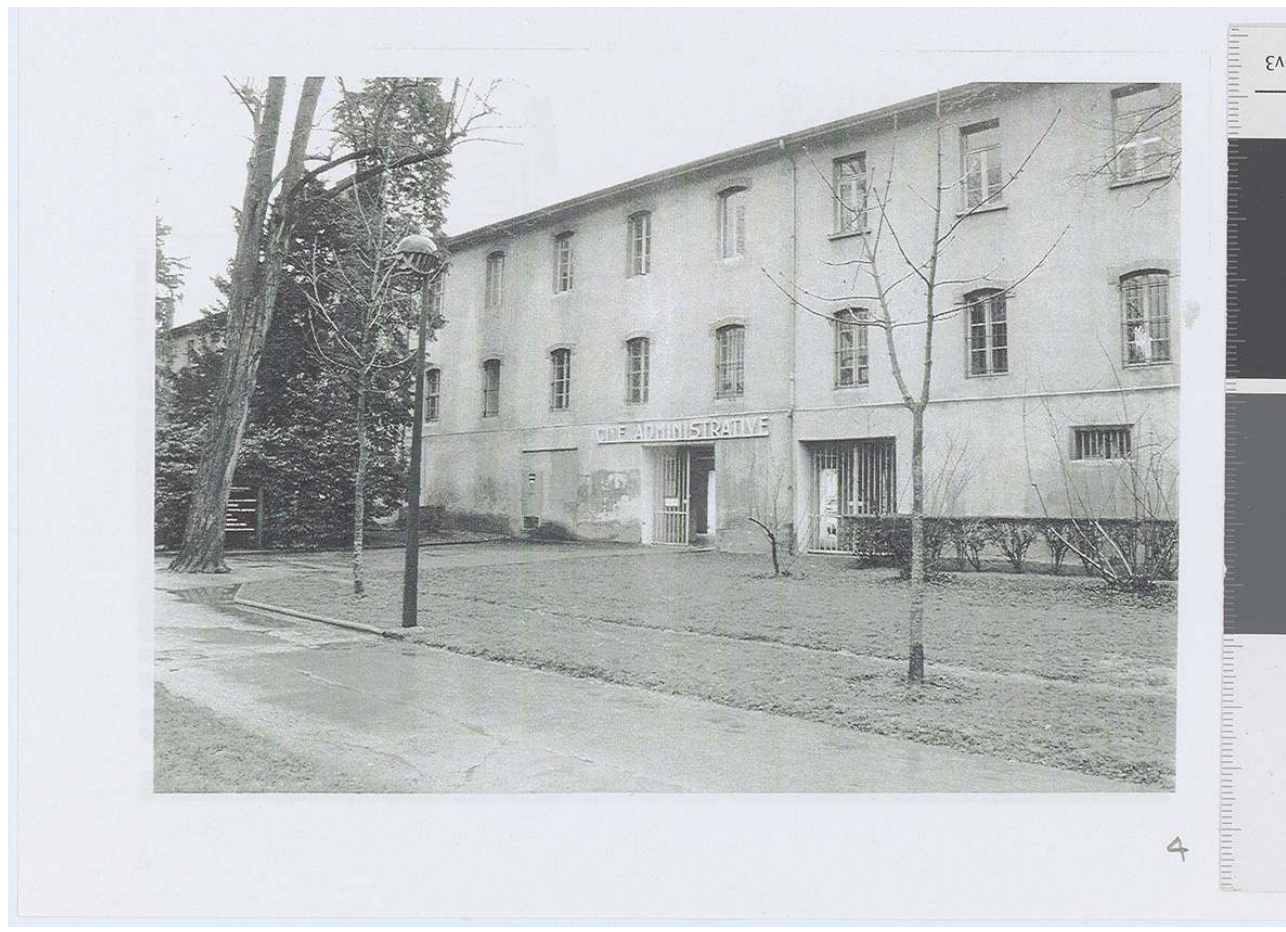

Vue de l'un des bâtiments (probablement le bâtiment $\mathrm{H}$ ) de la cité administrative de Nancy, tel que donné en illustration en 1995 dans le dossier du projet de réaménagement présenté dans le cadre du programme «EUROPAN 4 ».

(C) Archives nationales, 20160020/911. 


\section{Bordeaux : le choix de la hauteur}

13 Comme dans toutes les grandes villes de France, le projet de construire à Bordeaux un ou plusieurs bâtiments destinés à regrouper tous les services publics s'est très tôt imposé après la guerre. Contrairement à Nancy, les orientations ont été ici assez rapidement fixées et ont pu être menées à leur terme dans un délai qui peut paraître raisonnable compte tenu de l'ampleur du projet et du nombre d'acteurs impliqués.

Peu après la Libération était apparu à Bordeaux un projet de regroupement des services administratifs en un seul lieu. Les études menées en ce sens orientèrent le choix du terrain (qui impliquait d'être suffisamment vaste, disponible et accessible depuis la ville) sur celui dit de l'American Park, du nom d'un parc d'attractions qui y était en activité au début $\mathrm{du} \mathrm{xx}^{\mathrm{e}}$ siècle. La Commission centrale de contrôle des opérations immobilières approuva le projet le 20 janvier 1950 et par arrêté du 24 octobre suivant, l'architecte en chef des Bâtiments civils et Palais nationaux Pierre Mathieu était choisi ; un tiers de la superficie du terrain pour une durée de cinq ans fut réservé par décret du 26 avril 1951. En novembre 1954, alors que le financement restait à définir, un " programme officieux » était présenté par Mathieu devant le Conseil général des bâtiments civils, suscitant diverses remarques relatives à l'accessibilité, aux liaisons et à l'équilibre entre les bâtiments composant l'ensemble ${ }^{21}$. En 1955 le principe de l'édification d'une cité administrative était approuvé par la Commission des opérations immobilières et à la fin de cette même année le terrain de l'American Park était acquis par l'État : la mise en état préalable au chantier fut aussitôt entreprise. Cependant, en 1957, le conseil municipal de Caudéran (commune sur laquelle se trouvait le terrain) se déclarait opposé à ce projet, entrainant l'interruption des travaux préparatoires et une inspection diligentée par le ministère des Finances. À la recherche d'une solution alternative, le préfet de la Gironde informait en février 1958 la Commission des opérations immobilières qu'un entrepôt inoccupé, situé en centre-ville et appartenant à la chambre de commerce de Bordeaux, pourrait être mis à la disposition de la Ville pour y reloger les services administratifs alors installés de manière précaire. Après une première étude commandée à l'architecte Mathieu, la direction de l'Architecture du ministère de l'Éducation nationale envoya sur place en 1959 l'inspecteur général André Leconte, qui confirmait dans son rapport l'avis assez défavorable de Mathieu concernant cette réutilisation de l'entrepôt: si l'idée d'aménager ce bâtiment du XVIII ${ }^{\mathrm{e}}$ siècle pour un usage administratif pouvait "paraître à première vue séduisante et même économique ", dans la pratique les travaux de remise aux normes aboutiraient à un coût extrêmement élevé sans pour autant obtenir un résultat satisfaisant; il lui semblait donc plus judicieux de s'orienter vers une nouvelle construction :

En résumé, on aurait refait les planchers, reconstruit des murs nouveaux, et il resterait par dessus le tout un mauvais plan avec quatre angles morts parfaitement impropres pour un bâtiment administratif. [...] En ce qui concerne l'American Park, la solution proposée est certainement de plus grande envergure, garantissant mieux l'avenir. Elle permet des solutions architecturales plus osées, des bâtiments mieux aérés. Il est clair que l'architecte sera plus à l'aise sur un terrain de trois hectares et demi que sur un terrain d'un demi-hectare ${ }^{22}$.

Après divers échanges opposant le ministère des Finances, la direction de la Construction, la municipalité de Bordeaux et la préfecture, la Commission des opérations immobilières 
envoya à son tour en 1960 une inspection qui se montra favorable à une construction sur le terrain de l'American Park:

Si à la place des entrepôts Vauban et Lainée ${ }^{23}$, il y avait eu un terrain immédiatement disponible, cet emplacement, bien que permettant un regroupement moins important, eut sans doute été préféré à celui de l'American Park. En l'état actuel des choses, et tout en comprenant les efforts par les responsables locaux pour parvenir à la solution Vauban-Lainé, il y a lieu de retenir comme élément essentiel l'urgence des besoins à satisfaire, ce qui conduit à rechercher la solution la plus simple, la plus rapide. La solution American Park répond à ce souci, beaucoup mieux que celle Vauban-Lainé et elle est moins coûteuse. Il ressort des constatations faites sur place que le terrain, d'un accès, plus facile au moyen de véhicules individuels qu'au moyen de transports collectifs, ne peut être considéré comme très éloigné. [...]. Si la commission adopte cette proposition, il serait nécessaire que le programme puisse être réalisé rapidement [...]. La première fraction des travaux pourrait être entreprise dès le premier semestre 1961 au moyen des crédits disponibles, les dispositions nécessaires étant prises pour que le financement du surplus soit assuré à partir de $1962^{24}$.

De fait, l'architecte Mathieu présentait en juillet 1961 un nouveau projet devant le Conseil général des bâtiments de France, de nouveau évalué par André Leconte. Son appréciation était assez mitigée :

Le rapporteur observe que le parti d'un bâtiment unique en forme d'arc de cercle de $180 \mathrm{~m}$ de longueur développée, qui se construit tranche après tranche, présente sans doute des avantages de souplesse du point de vue de l'absorption des crédits, mais il n'en est pas moins discutable. Il constitue une sorte d'échelle dans cette zone située à Caudéran, limite de Bordeaux. Il se présente vers la ville dans sa partie convexe, alors qu'on eut mieux compris qu'il présentât sa partie concave précédé du parc jardin qu'on a l'intention d'établir. Il y a en réalité assez peu de dégagements autour du terrain pour justifier du recul qui sied à une composition aussi monumentale. On peut critiquer d'ailleurs la forme courbe en soi, elle n'est pas génératrice d'économie, bien au contraire. Elle est de plus difficile à exécuter. D'autre part, le plan-masse devrait s'appuyer sur un programme précis, avec des extensions possibles, et faire un état de tous les éléments prévus ou prévisibles [...]. En conclusion, le rapporteur propose au Conseil général des bâtiments de France qu'une nouvelle étude, basée sur un programme complet, soit demandée à l'architecte [...]. Ces observations sont portées à M. Mathieu introduit en séance. Celui-ci fait savoir qu'il s'agit d'un projet déjà ancien, établi à partir de données datant de 1954 et qui a été augmenté pour tenir compte de demandes successives de locaux supplémentaires. Il précise à cet égard qu'il n'a pu, du fait des modifications incessantes du programme, établir un projet répondant à des besoins précis [...]. M. Mathieu indique par ailleurs qu'en accord avec les observations qui lui sont présentées sur la conception architecturale, il abandonnera le principe d'un vaste bâtiment courbe pour adopter une composition comportant une partie verticale nettement affirmée, accompagnée de bâtiments plus bas qui s'harmoniserait avec le caractère de la ville ${ }^{25}$.

17 Le nouveau projet de Pierre Mathieu, soumis moins d'un an plus tard au Conseil général des bâtiments de France, était en effet bien différent :

Dans son nouveau plan masse, M. Mathieu présente un parti de construction en hauteur. D'autre part, la réalisation étant prévue en deux tranches, l'architecte a conçu deux bâtiments parallélépipédiques implantés dans la partie nord-est du terrain, l'un devant l'autre avec le déboîtement nécessaire pour en assurer l'éclairage. Les deux bâtiments seraient reliés par une galerie de jonction. Ils sont orientés nord-sud, orientation recommandable à Bordeaux-Caudéran [...]. Le parti de construction en hauteur s'inscrit dans un programme d'immeubles tours qui doivent être édifiés à la périphérie de la ville à une distance sensiblement égale du 
centre [...]. Le rapporteur estime qu'il ne semble pas que dans ces conditions la silhouette de la cité administrative puisse paraître insolite [...]. Le rapporteur conclut en indiquant que l'avant-projet $\mathrm{n}^{\circ} 2$ constitue un nouveau parti de plan qui présente une nette amélioration par rapport au parti précédent. Il est clairement exprimé et correspond bien au programme de deux tranches successives de travaux ${ }^{26}$.

la Commission centrale des opérations immobilières, qui l'approuva dans sa globalité, avec quelques menues réserves ${ }^{27}$. Le projet fut également approuvé par le Conseil général des bâtiments de France dans sa séance du 7 février, rendant possible le lancement des travaux ${ }^{28}$, les seules recommandations expresses du Conseil étant de laisser les abords de deux tours futures libres de toute autre construction ultérieure, afin bien mettre en évidence leur verticalité, et de créer autour des espaces verts et un parking. La tour A (haute de 90 mètres) fut achevée en 1968, la tour B (72 mètres) vers 1974 (fig. 3).

\section{Figure 3}

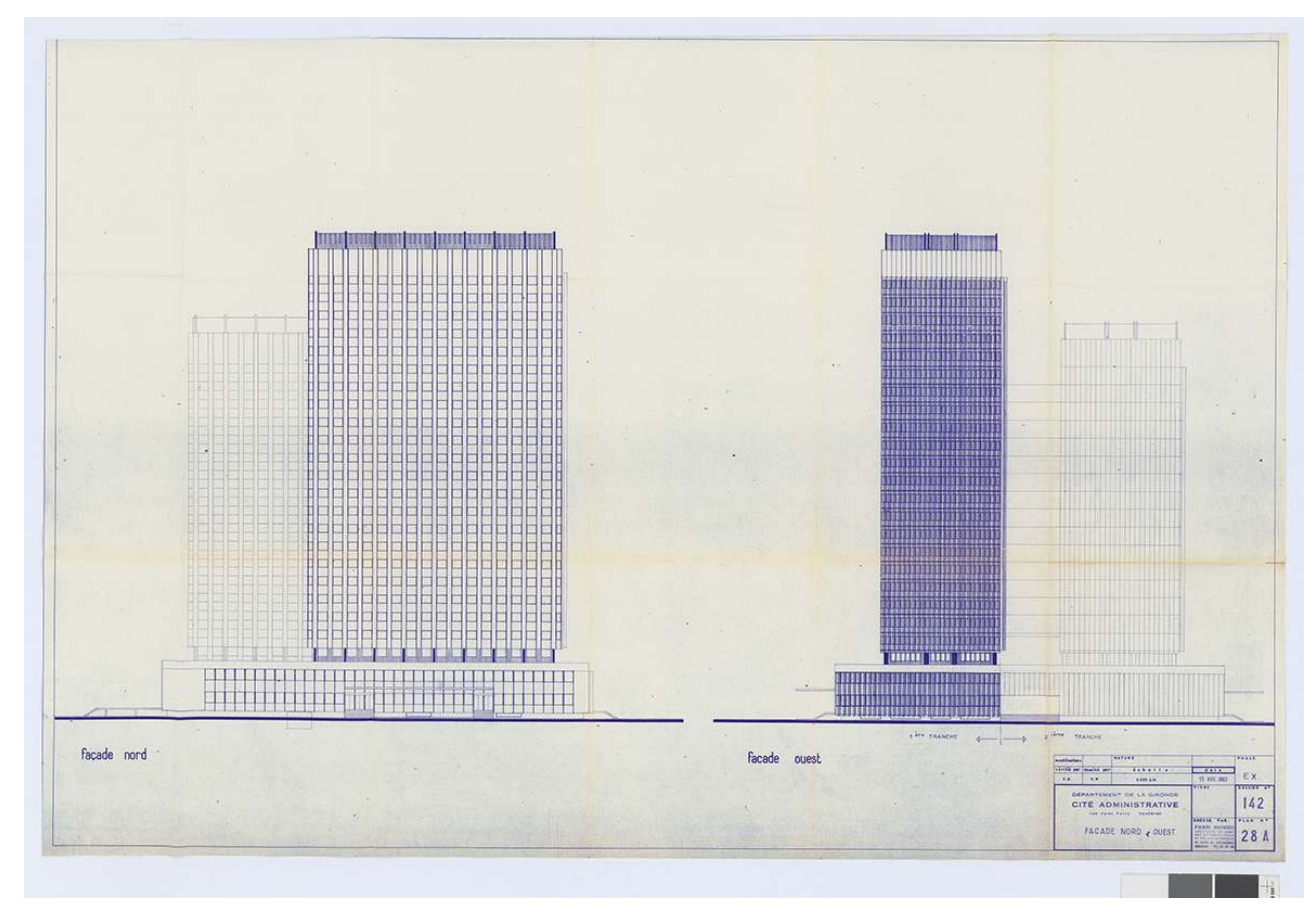

Cité administrative de Bordeaux. Façades Nord et Ouest. Pierre Mathieu, architecte en chef des Bâtiments civils et Palais nationaux (1963).

(C) Archives nationales, 19880557/49.

\section{Lyon : un ambitieux projet}

Le projet de regroupement des services publics à Lyon remonte au moins à 1954. En juin de cette année-là, le préfet du Rhône adressait à la CCCOI un avant-projet de plan de regroupement en sollicitant son accord de principe avant que soit engagée une étude approfondie du programme. Le rapporteur constatait qu'il ne s'agissait en fait que d'une 
concentration très partielle puisqu'il était proposé la construction de plusieurs bâtiments sur des sites parfois très éloignés les uns des autres : hôpital Desgenettes, cours Lafayette, rue Pierre-Corneille, rue Moncey et enfin, sur un terrain dépendant de la caserne de La Part-Dieu. Sur ce dernier, il était alors question de construire un hôtel de police ainsi qu'un «immeuble administratif » réunissant les services de différents ministères. En conclusion, la préfecture était invitée à étudier une exploitation plus rationnelle des terrains disponibles ${ }^{29}$.

21 En 1960 la CCCOI fut de nouveau consultée par la préfecture sur le projet de transfert au secteur civil de l'ensemble de l'ancien quartier militaire de La Part-Dieu et de l'ex-parc à fourrages. Dès son élection à la mairie de Lyon, Louis Pradel avait entrepris un grand plan de rénovation urbaine; une convention avait ainsi été signée avec la Société d'équipement de la région lyonnaise pour conduire ce nouveau projet d'aménagement. Le dossier présenté à la CCCOI rappelait qu'il existait déjà sur place une «cité administrative " réunissant quelques services publics et un centre d'hébergement de travailleurs nord-africains. Le programme immobilier prévoyait la construction de plus de 2000 logements ainsi que des groupes scolaires associés, un centre commercial, une maison de la radio, des espaces verts et une nouvelle cité administrative. Au cours des années suivantes, plusieurs plans se succédèrent sans pour autant aboutir ; ce n'est qu'en 1967 que le programme fut véritablement lancé, incluant bien sûr une nouvelle cité administrative. En novembre 1968 le préfet du Rhône Max Moulins adressait au Secrétariat général du gouvernement une note dans laquelle il présentait l'état d'avancement du dossier :

Le regroupement, dans une vaste cité administrative, de la plupart des services publics de l'État implantés à Lyon, a été envisagé il y a de nombreuses années, mais c'est seulement en 1967 que les études, conduites avec une certaine lenteur jusqu'alors, ont été reprises avec le souci d'une réalisation rapide. La raison essentielle de ce changement d'attitude a été la mise au point définitive, en 1967, du projet intéressant le centre directionnel de La Part-Dieu à Lyon. Cette opération de rénovation urbaine couvrant, en plein centre de la ville, 22 hectares [...] constitue, en fait, un point majeur de l'action d'aménagement du territoire dans la métropole lyonnaise et se trouve, dès à présent, en pleine réalisation, un certain nombre de bâtiments sont déjà achevés. Tout récemment M. Joël Le Theule [secrétaire d'État à l'Information] a inauguré la nouvelle maison de l'ORTF édifiée à La Part-Dieu ${ }^{30}$.

L'urgence était d'autant plus grande, selon le préfet, qu'en vertu de la loi du 31 décembre 1966, un certain nombre de communes de l'Ain et de l'Isère avaient été rattachées au département du Rhône, générant un fort accroissement d'activité pour les services administratifs départementaux. L'architecte Jean-Maurice Lafon avait été chargé de sa conception. Une première étude (datée du 17 avril 1968) comportait une tour de trente et un étages plus six bâtiments cubiques de six étages, l'ensemble devant être construit par tranches successives. Le projet, présenté devant le Conseil général des bâtiments de France le 4 juillet 1968, avait été validé sur son principe. Des réserves, tant sur la place disponible que sur le coût de ce projet, amenèrent l'architecte à proposer un plan très différent prévoyant huit bâtiments carrés de 32 mètres de côté, de hauteur allant de trois à vingt étages ${ }^{31}$. La réflexion était encore en cours l'année suivante. En effet, comme le signalait l'administrateur civil de la direction de l'Architecture, Sylvianne Grange :

Au cours de sa séance du 14 mai [1969], la Commission centrale de contrôle des opérations immobilières a examiné différents problèmes relatifs à la construction de la cité administrative de Lyon. Elle a notamment retenu le principe de l'application du système " espaces fonctionnels » pour la première tranche de cette 
cité destinée à l'ensemble des services financiers. La commission a reconnu que l'application de ce système impliquait une étude plus poussée du programme. Le S.C.O.M. doit se charger de cette étude de programmation en se faisant assister, par contrat, d'un programmeur extérieur (bureau Interorg en principe) ${ }^{32}$.

23 bien encadrer les prérogatives et domaines d'action de la société Interorg, chargée de la programmation. Dans ses notes, Claude Robin, sous-directrice de l'Architecture, écrit à propos du contrat à passer avec cette société: «Je ne vois pas quelle critique architecturale peut exercer le bureau d'études : c'est à la C.A. [la sous-direction de la Création architecturale] de jouer ce rôle, s'il est nécessaire, avant de passer le dossier en commission régionale ${ }^{33}$. »

Le compte rendu de la séance de la commission régionale Rhône-Alpes des opérations immobilières, de l'architecture et des espaces protégés du 10 mai 1971 indique que si le projet avait avancé, il était encore loin d'être abouti. Le rapport dressé par Maurice Novarina, architecte-urbaniste BCPN, nous livre une intéressante synthèse de l'état du projet :

[La CCOI] émit le vœu de voir étudier la possibilité d'installer les services en « espaces fonctionnels » ou «bureaux paysage ». Le but à atteindre était nouveau et, pour ce faire, une procédure inhabituelle fut adoptée. C'est ainsi qu'un bureau d'études spécialisé a été chargé d'analyser les besoins spécifiques d'une telle installation. Les hypothèses résultant de ces études ont été soumises à un collège de coordination chargé de confronter les critères de choix et d'arrêter les conclusions devant conduire au rapport final de synthèse. Ce rapport de synthèse est donc le document de base pour la conception du projet de la cité administrative ainsi que pour sa réalisation. L'architecte choisi pour cette opération est M.J. M. Lafon, architecte en chef des BCPN, 25, rue Spontini à Paris. L'architecte s'est attaché à suivre rigoureusement le programme de répartition des surfaces et des services dans l'espace et dans le temps. Il est en effet nécessaire de regrouper les services dans un certain ordre d'urgence et d'affinités. De plus, la construction d'un tel ensemble ne peut être envisagée qu'en plusieurs étapes, d'où la répartition en tranches physiques fonctionnelles. [...]. Programmation: Le programme établi par le bureau d'études spécialisé Interorg conduit à une surface HO de $119000 \mathrm{~m}^{2}$, extensions comprises, soit un léger dépassement de l'ordre de $13 \%$. L'ensemble de l'opération s'élèvera, y compris sous-sols, à $167500 \mathrm{~m}$. En accord avec les divers affectataires, le principe d'utilisation retenu est celui des «espaces fonctionnels » par plateaux de 2500 à $3000 \mathrm{~m}^{2}$ environ. Suivant les nécessités, quelques bureaux isolés pourront se situer au droit des façades en éclairage direct. Composition du projet: Il s'agit plus d'une esquisse poussée que d'un avant-projet. Le parti comporte un rez-de-chaussée général percé de deux grands patios, sur le périmètre duquel s'élèvent quatre bâtiments de hauteur différente : A : sur la rue Servient, $R+4$ sur pilotis; B : sur la rue Garibaldi, R+11; C : sur la rue Mazenod, R+8; D : vers la mitoyenneté, $R+3[\ldots]$. Mode de construction : L'ossature sera en béton armé. Les façades du plateau du rez-de-chaussée seront revêtues en pierre. Les superstructures seront en béton moulé. Les menuiseries seront en aluminium anodisé et les allèges en glace trempée émaillée [...]. Analyse du projet : compte tenu d'une programmation bien précisée dans l'espace et dans le temps, l'intégration des volumes, leur échelle, leur composition nous paraissent bonnes. Le jeu, la finesse, la qualité et la couleur des modénatures ajoutent à ces volumes simples une vibration nécessaire et heureuse. Cependant, la modénature générale du socle des bâtiments à rez-de-chaussée sur rues pourrait être encore améliorée. Le parti, fonctionnel et simple, est bien étudié. L'accueil est généreux et bien placé. La disposition des espaces autour de deux patios intérieurs est intéressante et offre beaucoup de possibilités surtout pour aménagement des bureaux fonctionnels ${ }^{34}$. 
Quel était donc ce concept d'« espace fonctionnel ", mentionné à plusieurs reprises dans

le rapport de M. Novarina? Jean Coussirou, secrétaire général-adjoint du Rhône, l'explicitait à sa suite :

Cette notion est encore très nouvelle. Il s'agit en fait d'opérer un changement radical dans les errements administratifs actuels, car le fait de passer d'un bureau de type traditionnel au bureau organisé en espace fonctionnel entraîne un certain nombre de conséquences importantes dans les méthodes de travail et dans les relations humaines à l'intérieur de chaque service. Il s'agit non de bureaux collectifs mais de plateaux de travail organisés et meublés de telle façon que chaque section d'un service fonctionne d'une manière autonome, tout en gardant des liens fonctionnels avec les autres sections. Le principe de base est de faire profiter tout le monde de l'espace total. Le volume est partiellement cloisonné jusque à la hauteur de $1,40 \mathrm{~m}$ environ par les meubles de rangement, ce qui permet aux personnes assises d'être isolées partiellement de la vue, et aux personnes debout et en déplacement de profiter de la perspective générale. Trois éléments essentiels sont traités de façon parfaite: l'acoustique, l'éclairage et la climatisation, de façon à créer une ambiance particulièrement attrayante. Plusieurs bâtiments administratifs sont actuellement construits en France suivant le principe de l'espace fonctionnel (Le Mans, Dijon, Nantua). Des visites sur place ainsi que des études précises permettent d'avancer que les avantages de l'espace fonctionnel sont les suivants : sur le plan humain: ambiance de travail excellente; meilleur rendement des fonctionnaires; fatigue moindre; conditions de confort plus adaptées aux exigences modernes;

sur le plan fonctionnel: utilisation optimale des surfaces; meilleures relations entre les services et interservices; polyvalence des locaux permettant des changements faciles en cas de restructuration des services;

sur le plan financier : économie de surface ; économie dans les frais de gestion; coût de construction moins élevé ${ }^{35}$.

Si l'aménagement en plateaux était alors apparemment nouveau en France, l'attrait de la hauteur était déjà très présent depuis plusieurs années. Lors de la discussion qui suivit ces exposés, Louis Pradel, maire de Lyon, regretta que les bâtiments proposés ne soient pas plus élevés : «On ne voit jamais assez grand, au départ ». Mme Grange, l'administrateur civil, rappela alors que le premier projet présenté par Jean-Maurice Lafon (en avril 1968) prévoyait justement une très haute tour, mais que l'idée avait été finalement écartée, en raison du surcoût et de la difficulté de fractionner les travaux en plusieurs tranches ${ }^{36}$ (fig. 4). 


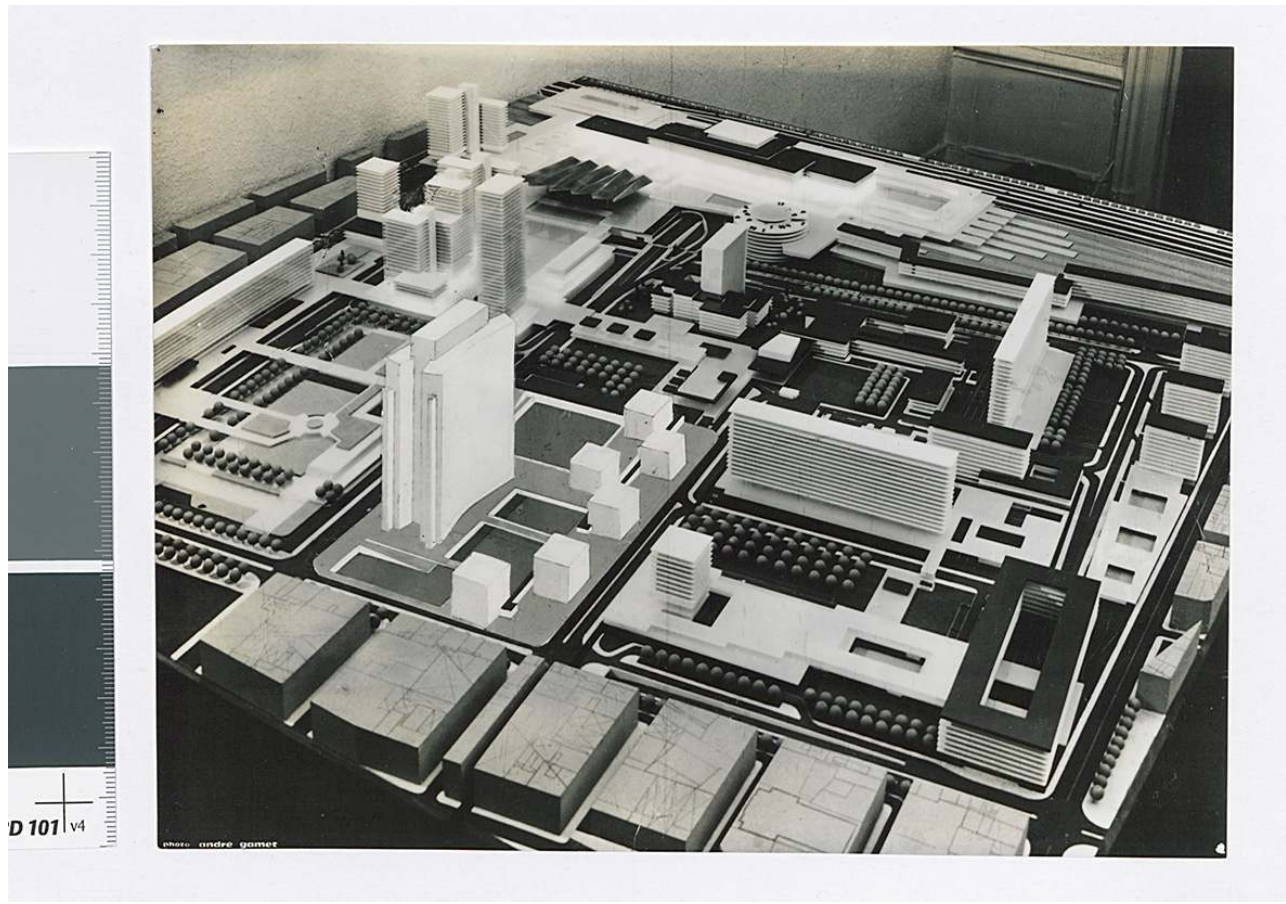

Cité administrative de Lyon La Part-Dieu. Photomontage sur la maquette générale de La Part-Dieu. J.M. Lafon, architecte en chef des Bâtiments civils et Palais nationaux.

Phot. Chevojon. (c) Archives nationales, 20060204/9.

Les travaux devaient débuter fin de l'année 1972. Dans ce contexte, un colloque national intitulé «Les cités administratives » fut organisé à Lyon les 25 et 26 mai 1972, sous la présidence inaugurale de Max Moulins, préfet de la région Rhône-Alpes, afin de susciter une vaste réflexion sur la place et la fonction de ce type de bâtiment au sein de la société ; il faisait aussi figure de bilan, au terme d'une vingtaine d'années de construction de ce type d'édifices dans toute la France. Le directeur de l'Architecture, Alain Bacquet, qui évoqua le «rôle du ministère de Affaires culturelles dans la réalisation des cités administratives » y voyait une « occasion directe d'architecture » :

Les constructions publiques, et notamment les cités administratives occupent une place importante dans la ville. Importante par leurs dimensions, par leur rôle et par les effets induits qu'elles entraînent alentour, sur l'organisation du tissu urbain et sur les circulations qu'elles provoquent. On peut dire d'une certaine manière que les constructions publiques sont des éléments structurants de la ville. L'État ne construit plus de palais, il construit rarement de nouveaux ministères. Les constructions publiques représentent pour la puissance publique une occasion directe d'architecture [...]. Jusqu'à un passé récent, la plupart des équipements administratifs exprimaient la volonté de puissance des pouvoirs qu'ils abritaient ou qu'ils représentaient, coupés d'un tissu urbain auquel ils ne s'intégraient pas, mais qu'ils dominaient, étrangers par leurs volumes, leurs matériaux et leurs techniques aux autres constructions. [...] Les constructions publiques connaissent une évolution qui développe tout à la fois les préoccupations d'ordre fonctionnel et d'ordre social [...]. La qualité plastique découle essentiellement de la qualité et de l'équilibre des différentes réponses données à des problèmes qui ne sont pas d'ordre plastique. La part de liberté en quelque sorte arbitraire de l'architecte créateur ne peut s'inscrire qu'à partir de ces contraintes et de ces solutions [...]. Mieux concevoir la cité administrative n'est donc pas seulement de bonne 
administration au niveau de l'outil qu'elle représente fonctionnellement parlant, c'est aussi concourir à la qualité de l'environnement urbain. À une époque où les pouvoirs publics s'inquiètent de jour en jour davantage de pollutions et d'environnement, améliorer cette qualité, c'est un peu favoriser autour de la cité administrative, la qualité de la cité elle-même, et la qualité de la vie ${ }^{37}$. également mise en œuvre pour accompagner les débuts de ce projet ${ }^{38}$. La première tranche des travaux devait s'achever en 1975, la troisième tranche devait se dérouler entre 1980 et 1985. Mais entre le retrait de certaines administrations afin d'échapper au regroupement initialement prévu et le désengagement progressif de l'État, le projet ne fut pas entièrement réalisé comme planifié. Charles Delfante, l'architecte chargé de la coordination du « centre directionnel de La Part-Dieu », interviewé entre 1999 et 2000, ne cachait pas son amertume et ne mâchait pas ses mots :

Tout le monde nous a reproché la surface de la cité administrative mais c'est là où l'État n'a pas joué le jeu! On fait la cité administrative, il y a un terrain de libre et la DASS se met ailleurs, rue Servient ; la DDE s'installe à La Part-Dieu mais ne lâche pas la rue Moncey, la DRAC ne vient pas, etc. alors qu'il y a une partie du terrain destinée à la cité administrative encore disponible! [...] Je crois que d'énormes problèmes juridiques se sont posés et qui n'ont pas été résolus. Pour deux raisons : d'abord parce qu'il n'y avait pas de législation et d'autre part parce qu'il n'y avait pas de jurisprudence! [...]. Comment une société d'économie mixte, comment des architectes en chef, comment un président de communauté urbaine peuvent-ils influer sur une décision prise directement par un ministère? Si les règlements ne sont pas respectés, si les objectifs du projet sont battus en brèche, ça ne fait rien! C'est pour ça que l'on a la Cité administrative d'État, « tombée du ciel »! Qui aurait dû dépasser les coefficients d'emprise et les coefficients d'occupation du sol ? L'État a changé son fusil d'épaule, plus exactement les différents services de l'État ! Si un directeur de n'importe quel service d'État dit: "Je ne veux pas aller à la PartDieu ! " et qu'il le démontre à son ministère, que pour mille et une raisons de coût, de commodité, etc. qu'est-ce que vous faites ?39

Il avait vu le projet lui échapper peu à peu, entre querelles politiciennes, volonté d'économie et de rentabilité et résistances administratives. Alors qu'il avait imaginé un vaste plan d'ensemble, au bout de quelques années, les constructions furent confiées par lots à divers organismes (publics et privés) qui prirent de plus en plus de liberté avec le programme initial, le privant définitivement de la cohérence prévue sur le papier. Le quartier de La Part-Dieu fait aujourd'hui l'objet d'un nouveau projet urbanistique et il est question que les sept bâtiments de la cité administrative soient vendus pour être réhabilités en vue de nouvelles activités.

Les trois exemples retenus pour cette étude sur l'histoire de la construction de cités administratives, vue au travers des archives de l'administration des Bâtiments civils, dénotent non seulement des parcours et des résultats distincts les uns des autres mais illustrent aussi la complexité, et souvent la longueur de réalisation de ces projets architecturaux. Compte tenu du nombre et de la diversité des interlocuteurs - dont les objectifs pouvaient être notablement divergents -, on comprend qu'il ait parfois été long et difficile de s'entendre sur un projet donné. Si, théoriquement, la procédure semblait assez simple (formulation d'un projet au niveau local/préfectoral puis validation par les instances nationales - CGBC et CCCOI), dans la réalité, ces projets connurent souvent de nombreuses vicissitudes. Les collectivités locales, pressées par les administrations à installer ou à reloger, cherchaient des solutions rapides et pas trop onéreuses; la Commission centrale de contrôle des opérations immobilières devait accorder la 
rationalité et l'adéquation aux besoins avec une vision à long terme censée prévoir le développement urbanistique et administratif ; quant à la direction de l'Architecture, elle se devait de favoriser une création architecturale à la fois innovante et en phase avec son époque. Les circonstances (locales, politiques, administratives, etc.) et la réalité budgétaire eurent malheureusement bien souvent raison des objectifs et des idéaux initialement formulés par les uns ou les autres de ces acteurs. Outre les manœuvres de contournement opérées par certaines administrations afin d'échapper à un « exil » loin du centre ou bien dans un bâtiment (ou un quartier) jugé peu accueillant par leurs agents, qui pouvaient contribuer à nuire à la cohérence d'un plan d'ensemble initial (comme le soulignait Charles Delfante dans le cas de La Part-Dieu à Lyon), les trois exemples évoqués ici illustrent quelques-unes des grandes questions qui animèrent les débats autour de la construction de ces cités administratives dans toute la France. Face aux mesures d'économie ou aux préconisations de respect des budgets accordés avancées par certaines administrations, d'autres (par exemple à la sous-direction de la Création architecturale ou bien parmi les milieux politiques locaux) voyaient beaucoup plus grand, plus audacieux, plus «visible»; ce qui a pu favoriser l'émergence de projets monumentaux. On a vu comment le projet trop modeste initialement formulé par un architecte nancéien en 1968 est balayé par le directeur de l'Équipement ainsi que par le directeur de l'Architecture lui-même. L'époque était à la hauteur, aux formes originales, aux expérimentations: les constructions publiques ne pouvaient y échapper totalement. L'exemple lyonnais montre aussi l'émergence d'un parti pris d'aménagement alors nouveau, la création de plateaux ouverts (les fameux « open spaces » qui feraient ensuite fureur dans nombre d'immeubles de bureaux). Ce concept paraissait devoir s'imposer tant ses avantages étaient - un peu hâtivement ? - affirmés : « ambiance de travail excellente ; meilleur rendement des fonctionnaires; fatigue moindre; conditions de confort plus adaptées aux exigences modernes; utilisation optimale des surfaces...». Il fallait forcément le recul du temps pour faire la part des choses dans ce mode d'aménagement des espaces. La création de ces cités administratives était également soumise au débat centre/périphérie. Fallait-il rester en centre-ville? Ces quartiers centraux, pour faciles d'accès qu'ils fussent, présentaient souvent le désavantage d'être souvent densément construits, donc peu aisément extensibles ou modulables ; l'habitat y était dans bien des cas vétuste et donc répulsif aux yeux des aménageurs adeptes de la «modernité ». Le développement de l'urbanisme invitait d'un autre côté à repousser les frontières traditionnelles de la ville et par conséquent à considérer la périphérie comme une zone à conquérir puis à intégrer administrativement; l'implantation sur place de nouveaux services publics s'imposait donc. Bordeaux fit rapidement le choix de la périphérie, Lyon opta pour un ambitieux réaménagement du centre, Nancy envisagea (sans finalement pouvoir aboutir dans ses projets) d'établir un centre administratif dans son centre historique et dans un nouveau quartier extérieur. Enfin, la justification même de ces cités administratives fut parfois mise en question : on se souvient des débats houleux ayant eu lieu à Nancy en 1968, lorsque le directeur départemental de l'Équipement s'interrogeait sur « la nécessité de ces cités dans trente ans ». Le problème actuel de désertification de nombreux centres-villes relance aujourd'hui le débat sur le manque de commerces et de services de proximité, de déshumanisation de l'urbanisme, etc., ce qui devrait conduire les différents acteurs de l'aménagement du territoire à s'interroger sur les projets à entreprendre pour penser la ville (et sa relation avec sa très large périphérie - $\mathrm{y}$ compris rurale) des prochaines décennies. Les archives offrent pour cela la possibilité d'analyser 
avec le recul du temps les différents choix qui ont pu s'opérer en matière de construction publique - ici, avec le cas des cités administratives.

\section{NOTES}

1. - Voir par exemple l'ordonnance $n^{\circ} 45-2715$ du 2 novembre 1945 tendant à faciliter le regroupement des locaux administratifs dans la région parisienne (voir aux Archives nationales le versement 19770912: services communs urbanisme et transports; direction de l'Administration générale).

2. - En fait cette instance prenait son origine d'une première commission créée par arrêté du 12 août 1942 chargée de statuer sur l'opportunité des expropriations poursuivies par les administrations ; cet arrêté fut ensuite abrogé pour être remplacé par un décret de l'État français du 3 février 1943 instituant une Commission de contrôle des opérations immobilières, avant que lui soit substitué le décret du 2 novembre 1945. Cette nouvelle commission était placée sous l'autorité de la présidence du gouvernement provisoire et son travail était articulé avec des commissions départementales. Avec le décret du 28 août 1949 elle prend le nom de «Commission centrale de contrôle des opérations immobilières ", avant d'être remplacée par la Commission nationale des opérations immobilières et de l'architecture par décret du 28 octobre 1969. Sur la création de la Commission voir aux AN l'article 19820691/2 (direction de l'Aménagement du territoire ; CCOI).

3. - Par exemple à Amiens, Angers, Aurillac, Bar-le-Duc, les services administratifs furent provisoirement installés dans des locaux appartenant à l'armée (AN, 19880557/47, direction de l'Architecture ; sous-direction de la Création architecturale et des Constructions publiques ; cités administratives, correspondances relatives aux travaux à effectuer dans différentes cités (1954-1976)).

4. - Extrait du PV de la séance de la Commission centrale de contrôle des opérations immobilières (ci-après CCCOI) du 8 septembre 1950 (AN, 19910707/75, dossier « Nancy »).

5. - Extrait du PV de la séance de la CCCOI du 12 janvier 1951 (AN, 19910707/75, dossier «Nancy »).

6. - Commission centrale de contrôle des opérations immobilières ; dossier « cité administrative de Nancy » (1959) (AN, 19790874/3).

7. - Direction de l'Architecture ; sous-direction de la Création architecturale et des Constructions publiques ; cités administratives ; dossier « Nancy » (AN, 19880557/64).

8. - Bureau des Bâtiments civils; dossier « Nancy. Cité administrative » (AN, 19810663/901). On trouve dans ce dossier un premier rapport de Jean Bourgon daté du 30 octobre 1953, alors qu'il était déjà question du classement de la cité administrative de Nancy parmi les bâtiments civils. Par ailleurs le dossier contient les propositions de travaux (entretiens et grosses réparations) soumises par Bourgon, classées chronologiquement.

9. - Extrait du PV de la séance de la CDCOI du 15 avril 1957 (AN, 19910707/75, dossier « Nancy »). Ces deux bâtiments faisaient face au bâtiment $\mathrm{H}$, qui était le principal édifice déjà existant (voir plan accompagnant le PV de la réunion).

10. - « Rapport sur le projet de construction d'une cité administrative à Nancy » (8 avril 1965) (AN, 19900056/6: direction de l'Architecture; constructions publiques; dossier "cité administrative Nancy »). L'architecte La Mache sera de nouveau sollicité en 1963 et 1965 pour 
proposer d'autres solutions, tant dans le quartier central de l'ancienne caserne que sur le terrain périphérique d'Haussonville (voir ce même rapport).

11. - «Rapport fait au Conseil général des bâtiments de France» (20 septembre 1963) (AN, 19900056/6, direction de l'Architecture ; constructions publiques; dossier « cité administrative Nancy »).

12. - PV de la séance de la CCCOI du 24 mai 1964 (Ibid.).

13. - Note d'André Coumet, sous-directeur des Monuments historiques et des Sites (pour le ministre) au président de la CCCOI (2 novembre 1966) (Ibid.).

14. - PV de la séance de la CCCOI du 23 novembre 1967 (Ibid.).

15. - Note d'Yves Lormant au directeur de l'Architecture Max Querrien (19 juin 1968). André Laure reprochait notamment la monumentalité et la rigidité du projet de Roger Lamoise, qui aurait nui à toutes possibilités d'extension future (Ibid.).

16. - Note d'André Laure à Max Querrien, directeur de l'Architecture (Nancy, 22 juillet 1968) (Ibid $.$) .$

17. - Arrêté du 27 décembre 1968. Querrien avait quitté son poste de directeur de l'Architecture avant d'avoir eu le temps de signer l'arrêté mettant fin à la mission de Lamoise (Ibid.).

18. - Note de Jean Jenger au secrétaire d'État auprès du Premier ministre (9 juin 1972, ibid.). Quelques semaines auparavant, Jenger avait reçu Sirvin et Lamoise, préoccupés de voir la préfecture changer de nouveau d'avis pour donner la préférence à des opérations ponctuelles et immédiates sur le terrain voisin dépendant de la caserne Thiry. En outre, une note de l'un de ses subordonnés adressée à Jenger (courant 1972 ?) concluait par ces mots : «Bien que constituant l'un des projets les plus crédibles, la cité administrative de Nancy n'est pas prévue sur les budgets immédiatement à venir du chapitre des charges communes ». (AN, 19880557/47 ; dossier "Cité administrative, Nancy »).

19. - Sur le projet présenté en 1995 voir aux AN l'article 20160020/911 : secrétariat du Plan Urbanisme Construction Architecture; Europan 4, "Construire la ville sur la ville, transformation de sites urbains contemporains », dossier « Nancy, cité administrative » (1995).

20. - Je remercie vivement Bertrand Tonnelier, des archives départementales de Meurthe-etMoselle, des informations aimablement transmises en juillet 2016 sur le destin de ces projets architecturaux. Des documents complémentaires sur ces projets successifs sont bien sûr aussi conservés aux archives municipales de Nancy et aux archives départementales de Meurthe-etMoselle.

21. - En préambule à son compte rendu le président de la section spéciale des Bâtiments civils déclarait : «Il convient de n'attacher à cette étude établie sur la base d'un programme officieux qu'une valeur indicative » (Conseil général des bâtiments civils, PV de la séance du 18 novembre 1954, AN, F/21/6599).

22. - Rapport de l'inspecteur général André Leconte (Paris, 29 septembre 1959) (AN, 19810663, direction de l'Architecture, bureau de la Construction; dossier «cité administrative de Bordeaux »).

23. - Les entrepôts Lainé ne furent finalement pas retenus pour ce projet. Menacé de démolition, ce bâtiment fit l'objet d'une campagne de sensibilisation qui aboutit à leur inscription à l'inventaire supplémentaire des monuments historiques en 1973. Le Centre d'art contemporain s'installa dans une première partie de l'édifice en 1974, puis, en 1984, commença une nouvelle phase de réhabilitation qui s'est achevée en 1990.

24. - Rapport présenté par la sous-commission chargée d'une étude sur place (1960) (AN, 19790874, Secrétariat général du gouvernement, archives des chargés de mission Michel Lhuilier et Camille Bailly ; examen par la Commission centrale des opérations liées aux regroupements des services publics de province; dossier « Bordeaux »).

25. - Conseil général des bâtiments civils. PV de la séance du 27 juillet 1961 (AN, F/21/6599, direction de l'Architecture). 
26. - PV de la séance du 8 mars 1962. Le rapporteur était de nouveau André Leconte (Ibid.).

27. - PV de la séance de la CCCOI (7 janvier 1963). Les derniers mots sont soulignés dans le PV dactylographié (Ibid.).

28. - PV de la séance du CGBF (7 février 1963 ; ibid.). À noter qu'un jeu de plans du projet est conservé sous les cotes 19880557/49 et 50 (direction de l'Architecture; bureau de la Construction ; dossier « cité administrative Bordeaux »).

29. - Conseil général des bâtiments civils; PV de la séance du CCCOI du 3 décembre 1954 (AN, $\mathrm{F} / 21 / 6634)$.

30. - Note du préfet du Rhône au Premier ministre, (Lyon, sans date) [reçue au Secrétariat général du gouvernement le 20 novembre 1968] (AN, 197800874 : CCCOI ; dossier « Lyon »).

31. - Note de J. Maurice Lafon sur ses études préliminaires (sans date) (AN, 19880557/53, direction de l'Architecture ; bureau de la Construction ; dossier « cité administrative Lyon »).

32. - Note de S. Grange au directeur de l'Architecture (28 mai 1969) (Ibid.).

33. - Notes de Claude Robin, relatives au contrat Interorg à destination de Mme Grange, administrateur civil (17 mars 1971). Le mot était ainsi souligné par Robin (Ibid.).

34. - PV de la séance de la commission régionale Rhône-Alpes des opérations immobilières, de l'architecture et des espaces protégés du 10 mai 1971 (AN, 19880557/54, direction de l'Architecture ; bureau de la Construction ; dossier « cité administrative Lyon [suite] »).

35. - Ibid.

36. - Ibid.

37. - Des documents préparatoires à ce colloque (organisé par l'Institut technique des administrations publiques), émanant de la direction de l'Architecture, se trouvent aux AN sous la cote $19880557 / 47$.

38. - Voir un exemple de plaquette sur le projet de La Part-Dieu publiée au début des années 1970 (AN, 20060204/9 (bureau des Bâtiments civils)).

39. - Charles Delfante, entretiens avec l'équipe EDU-INSA, 1999-2000, cité dans AUTRAN, Stéphane. «Le quartier de La Part-Dieu à Lyon, l'invention d'un centre-ville, 1960-1980». Millénaire 3. Le centre Ressources Prospective du Grand Lyon, 2008, p. 22-23. Voir sur le site: http://www.millenaire3.com/content/download/3392/58270/version/1/file/

Quartier_Part_Dieu.pdf [consulté le 29/08/2016].

\section{RÉSUMÉS}

Après la Seconde Guerre mondiale, dans un contexte de reconstruction massive, de développement de l'urbanisation et d'évolution des services publics, la construction de cités administratives occupe une place prépondérante dans le paysage architectural et urbanistique de la France des "Trente Glorieuses ». Les archives des services des Bâtiments civils et de l'Urbanisme conservées aux Archives nationales permettent de documenter cette page de l'histoire architecturale. Si l'on observe des constantes dans la conduite des projets (de grands principes de développement urbain ; un allongement dans le temps souvent notable du suivi des projets; des allers et retours incessants entre l'échelon central et le niveau local), on constate aussi une grande diversité des approches, illustrant des ambitions tant politiques qu'urbanistiques ou architecturales, parfois antagonistes du fait de la diversité des acteurs impliqués ou du contexte local. 
After the Second World War, in a context of massive reconstruction, tremendous development of urbanisation and the evolution of public services, the building of administrative centres occupied a major place in the French architectural and town-planning landscape of this period of economic prosperity. The archives of architectural and town-planning administrations held by the National Archives (Pierrefitte) allow us to document this page of architectural history. If we can observe unchanging elements in the way the projects are conducted, we also notice a significant diversity in the approaches, illustrating ambitions that might be political, urbanistic or architectural. These approaches can sometimes be antagonistic because of the diversity of the actors involved or the local contexts.

\section{INDEX}

Mots-clés : France, Après-guerre, architecture, construction, cités administratives

Keywords : France, Post-war, architecture, construction, administrative centres

\section{AUTEUR}

\section{PASCAL RIVIALE}

Chargé d'études documentaires, Archives nationales, département Éducation, culture, affaires sociales (DECAS). Docteur en histoire, chercheur associé au centre EREA du Laboratoire d'ethnologie et de sociologie comparative (CNRS-Université de Paris-Nanterre) pascal.riviale@culture.gouv.fr 